\title{
Electronic and Geometric Structure of small mass selected Clusters
}

\author{
G. Ganteför, Chia-Yen Cha, H. Handschuh, G. Schulze Icking-Konert, B. Kessler, \\ O. Gunnarsson* and W. Eberhardt
}

Institut für Festkörperforschung der KFA-Jülich, D-52425 Jülich, Germany

\begin{abstract}
An experimental setup for photoelectron spectroscopy on mass selected cluster anions is described. The photoelectron spectra reveal the electronic structure of the particles as function of their size (mass). For clusters consisting of elements which are counted among the simple metals, i.e., the alkali's as well as $\mathrm{Cu}, \mathrm{Ag}$, and $\mathrm{Au}$, the electronic structure is indicative of extended, delocalized states formed by the atomic s-electrons. This is the first indication of metallic behavior even though the particles containing up to 20 atoms clearly exhibit a 'bandgap' in the electronic structure. Additionally, in cases where vibrational finestructure is observed in the photoelectron spectra, the measured vibrational quanta allow to deduce the particle geometry. This is especially helpful in studies of chemisorption phenomena on the clusters. Moreover, electron phonon coupling constants can be extracted from a high resolution spectrum of $\mathrm{C}_{60}{ }^{-}$, which confirm the alkali doped fullerenes to be conventional BCS type superconductors.
\end{abstract}

\section{Introduction}

Clusters are small particles having a well defined number of atoms. On the size scale they quasi-continuously bridge the gap from the atom to the three dimensional solid. From a materials science point of view their properties, however, develop in a noncontinuous fashion, leaving ample room for the existence of totally new materials. One example of such new materials are the fullerides $/ 1 /$, which are van-der-Waals bound solids consisting of $\mathrm{C}_{60}$ clusters. The fullerides have distinctly different properties than diamond or graphite, the other known forms of solid carbon.

The 'material properties' of the clusters are characterized by their geometry and their electronic structure. The electronic states determine the stability of the clusters and other properties such as whether the particle is metallic or magnetic and also how it interacts with an adsorbate molecule. One of the intrinsic questions asked, for example, is how many atoms are needed until metallic behavior occurs. This obviously also hinges on the definition of what constitutes metallic behavior and how it may be detected.

The possible novel materials properties of clusters may be conserved by depositing them onto a nonreactive substrate. If this can be done successfully, then technical applications as catalytic or electronic materials can be envisioned. The gas phase/molecular beam experiments, however, offer a very fast and convenient screening method for the detection of the cluster properties as a function of their size.

\section{Experimental setup}

High resolution photoelectron spectra of mass selected cluster anions /2/ can reveal

*Max-Planck-Institut für Festkörperforschung, D-70506 Stuttgart, Germany 
some of their characteristic properties. At low resolution the photoelectron spectra allow to determine the electronic structure, since the spectra show the various electronic transitions from the ground state of the anion into the ground and excited state configurations of the neutral system. High resolution spectra /3/ additionally may exhibit finestructure due to the excitation of vibrational quanta of the neutral cluster, as determined by the Frack-Condon overlap between the anion ground state and the selected electronic configuration of the neutral cluster. The vibrational frequencies thus observed are characteristic of the geometry of the particle.

Our experimental setup /2,3/ for photoelectron spectroscopy on mass selected cluster anions is shown schematically in Fig. 1. Mass selected clusters are produced by a pulsed arc cluster ion source (PACIS). The electric arc vaporizes the chosen material. The atoms and ions of the plasma

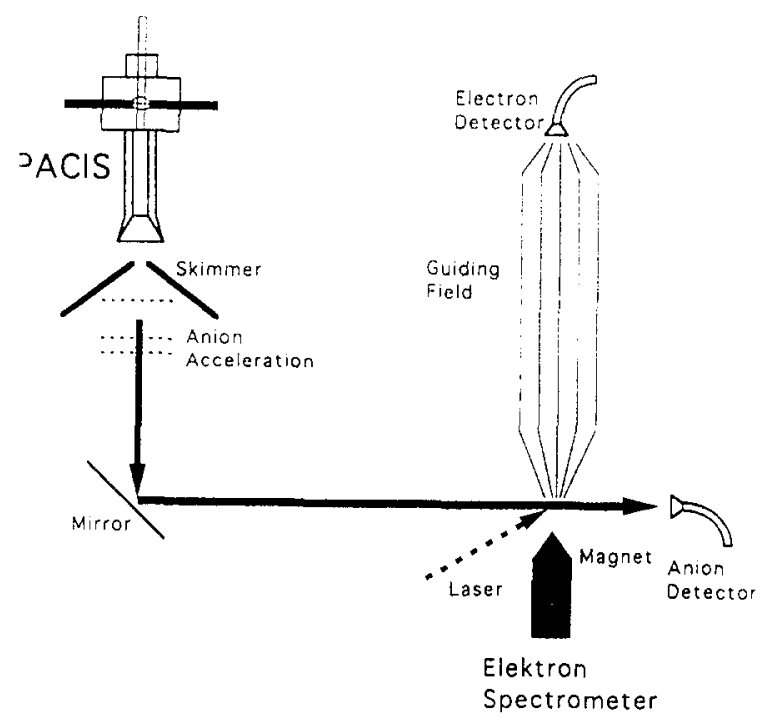

Fig. 1 Schematic of the experimental setup for photoemission spectroscopy of mass selected cluster anions. Shown are the pulsed cluster source (PACIS), the ion acceleration and deflection, and the magnetic bottle type time of flight electron spectrometer. are condensed into small aggregates in a high pressure inert gas atmosphere. Subsequently, a molecular beam is formed through adiabatic expansion, containing a distribution of non-interacting particles. The negative cluster ions within this beam are accelerated and deflected in a pulsed Wiley McLaren type time of flight setup. Photoemission (photodetachment) of electrons from cluster anions of a selected mass only is achieved by adjusting the time of the detachment laser pulse to the time of arrival of clusters of a certain mass at the source region of the magnetic bottle type time-offlight electron spectrometer /4/. In order to achieve an overall resolution of better than $10 \mathrm{meV}$, the Doppler broadening has to be reduced by decelerating the clusters prior to the photodetachment process.

\section{Results and Discussion}

\section{A. The electronic structure of simple metal clusters}

As mentioned above, the question of the onset and the development of metallic behavior is certainly one of the fundamental questions in the physics of clusters. One criterion is the existence of a finite size bandgap. This can be readily investigated in photoemission spectroscopy. As we will show later, none of the clusters investigated here have a vanishing bandgap.

Another indication of metallic behavior is the existence of electronic states which are delocalized throughout the whole particle. The eigenstates of a delocalized electronic system should be arranged in shells with well defined quantum numbers and degeneracies. The electronic states and the level structure for spherically symmetric particles are schematically indicated in Fig. 2 for a system containing 40 electrons 15/. The quantum numbers of the electronic states correspond to the number of radial and spherical nodes of the delocalized wavefunction. Accordingly a level sequence of $1 \mathrm{~s}(2), 1 \mathrm{p}(6), 1 \mathrm{~d}(10), 2 \mathrm{~s}(2), 1 \mathrm{f}(14), 2 \mathrm{p}(6) \ldots$ is predicted, whereby the numbers in the brackets denote the degeneracy of the corresponding shell. Obviously, if the 
spherical symmetry of the particle is broken, the degeneracy of these shells is lifted.

Relative abundancies and stabilities of alkali clusters as a function of their size have been explained using this electronic shell model $/ 5 /$. For example particles containing 20 or 40 valence electrons correspond to a cluster with a closed electronic shell and therefore should exhibit a larger stability than the clusters immediately following these $(21,41)$. This is indeed found in the mass spectra.

The proof of existence of delocalized electronic states in small clusters, which are arranged in electronic shells, was one of the first objects of our investigations $/ 6,7 \%$. A series of $\mathrm{Cu}$ spectra for $\mathrm{Cu}_{1}{ }^{-}$to $\mathrm{Cu}_{8}{ }^{-}$is shown in Fig. 3. In these spectra, taken at

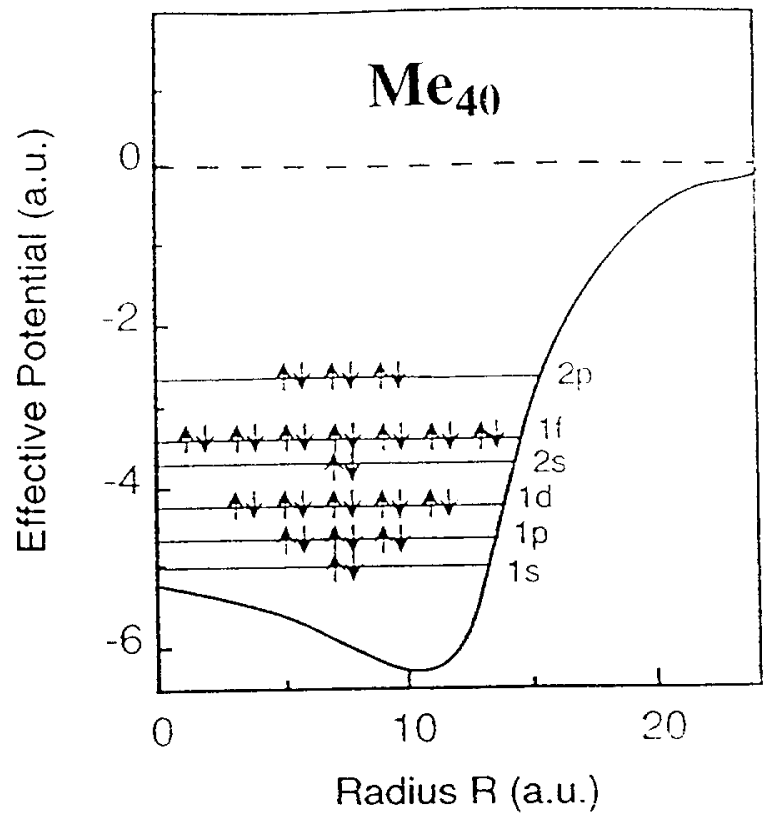

Fig. 2 Schematic of the energy levels including the occupancy of the delocalized electronic states within a spherically symmetric potential filled with $\mathbf{4 0}$ electrons /5/. $\mathrm{Me}_{4} \mathrm{O}$ denotes that this might be applicable to any 40 atom cluster of elements where the bulk solid exhibits metallic behavior.

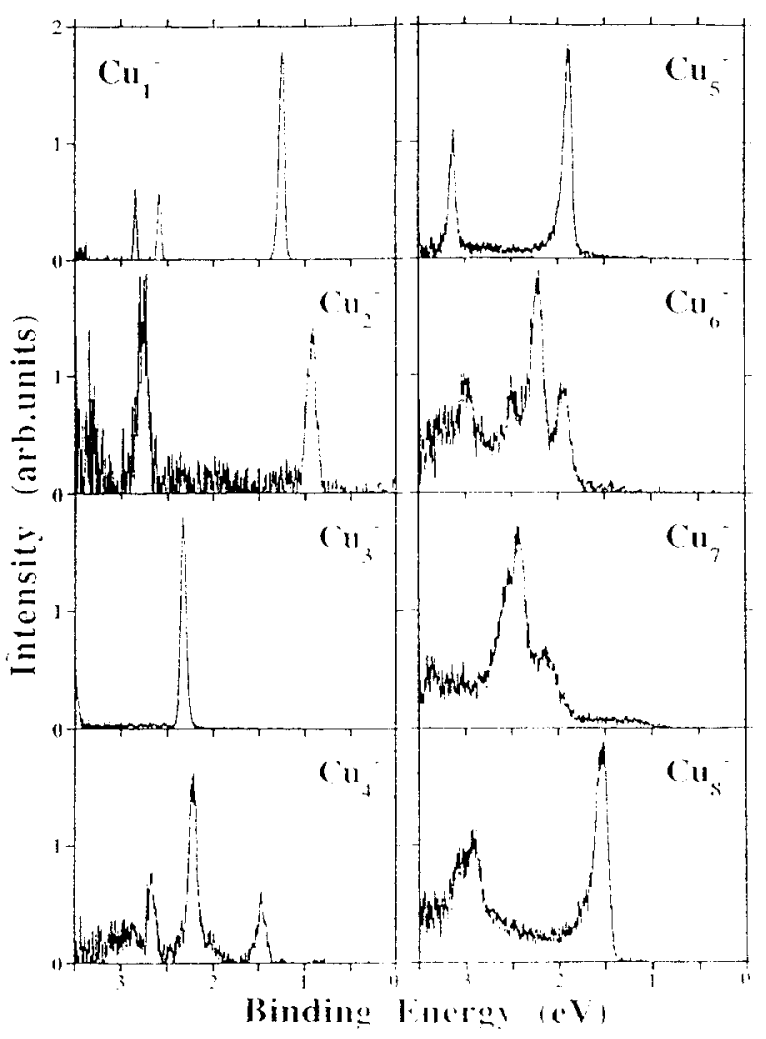

Fig. 3 Photoelectron spectra of mass selected, negatively charged $\mathrm{Cu}$ clusters taken with $\mathrm{N}_{2}$ laser radiation $(\mathrm{hv}=3.6 \mathrm{eV})$

$3.6 \mathrm{eV}$ photon energy, already the features of the electronic shell model are dominating the emission characteristics of the states which derive their character largely from the atomic $4 \mathrm{~s}$ states. Obviously the $\mathrm{Cu} 3 \mathrm{~d}$ electrons remain very localized and do not participate in the delocalized 'metallic' states. Their binding energy is increasing rapidly $/ 8 /$, such that within the binding energy range shown in Fig. 3 only the spectrum of the negatively charged atom exhibits two lines between 2.5 and $3 \mathrm{eV}$ binding energy, which are attributed to the $\mathrm{Cu} 3 \mathrm{~d}$ emission. Their energy separation corresponds exactly to the spin-orbit splitting of the $3 \mathrm{~d}_{5 / 2}$ and $3 \mathrm{~d}_{3 / 2}$ final core hole state. The strong feature at a binding energy of about $1.2 \mathrm{eV}$ is attributed to emission from the doubly occupied $4 \mathrm{~s}$ level. This spectrum thus also demonstrates that the emission cross section from $s$ derived states is much larger at these low 


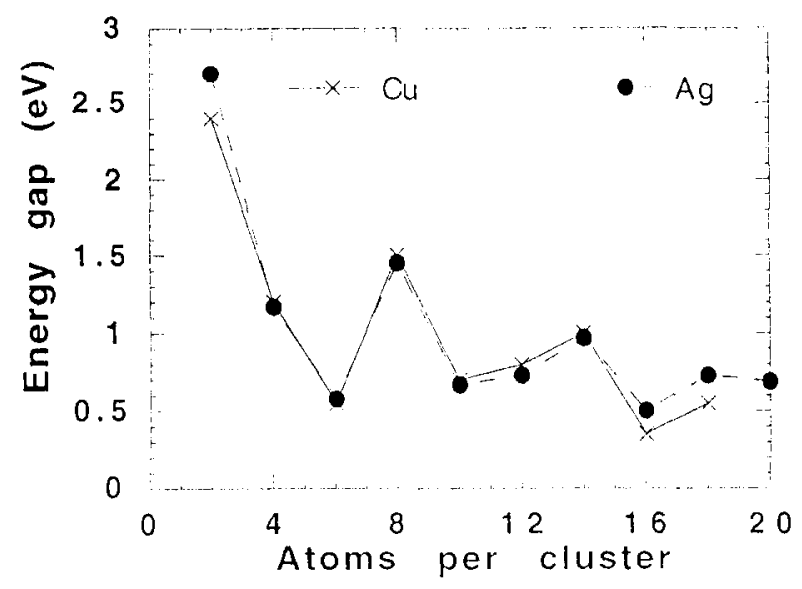

Fig. 4 The energy of the optical gap in small, negatively charged $\mathrm{Cu}$ and $\mathrm{Ag}$ clusters as a function of size. For the exact definition of the optical gap see text.

atomic $\mathrm{d}$ states, which have a degeneracy of 6 and 4, respectively.

The spectra of the larger clusters are dominated by the even/odd alternation $/ 5 /$ of the vertical detachment energy, i.e., the binding energy of the outermost electron of the negatively charged cluster. The vertical detachment energy (VDE) of the cluster anions differs from the electron affinity of the neutral cluster in cases where the geometries are different, due to the fact that within the Born Oppenheimer approximation electronic transitions are observed in the geometry of the anion $/ 9 /$. According to this alternation of binding energies, all the even numbered clusters, which are containing an odd number of electrons since they are negatively charged, have low VDE's and all the odd numbered clusters have higher VDE's.

In the negatively charged atom the s-shell is already completely filled. Accordingly, $\mathrm{Cu}_{2}{ }^{-}$ has three electrons in the delocalized states and these are occupying two shells. The smaller peak in the spectrum is attributed to the emission from the p-shell, whereas the emission of the s-shell gives rise to two peaks, separated in energy by the singlettriplet splitting of the final state. Only the peak attributed to the triplet final state is seen in Fig. 3. At larger excitation energies, however, the corresponding singlet state is observed as well $/ 6,7 /$. The spectra of $\mathrm{Cu}_{4}{ }^{-}$ and $\mathrm{Cu}_{6}{ }^{-}$clearly exhibit this splitting in the second emission feature. The singlet triplet splitting decreases with increasing cluster size as expected for more delocalized electronic states.

Continuing the interpretation of the spectra on the basis of the shell model, for $\mathrm{Cu}_{7}^{-}$the $\mathrm{p}$ shell of the cluster is completely filled, since this cluster contains 8 delocalized electrons. Accordingly, the next larger cluster, which now contains a dsymmetry delocalized electron, exhibits a clearly split off sharp peak at about $1.6 \mathrm{eV}$ binding energy.

Within this simple framework, the HOMO-LUMO gap of the neutral cluster, which in the solid state analog corresponds to the bandgap of the system, is directly measured in the spectra of the even numbered clusters. The energy separation of the lowest binding energy state, which in the final state configuration corresponds to the ground state of the neutral cluster, and the next higher singlet state is the HOMO. LUMO gap. Within this framework this corresponds to the first optically excited state since the creation of the triplet state is forbidden because it involves a spinflip transition. The values of the HOMO-LUMO gap energies thus derived for $\mathrm{Cu}$ and $\mathrm{Ag}$ clusters are shown in Fig. 4. Apart from a very general trend towards smaller values, there is fairly little systematic evolution in these energy gaps. Moreover, all small clusters investigated here are far from having a continuous density of states near the top of the occupied electronic states. In the solid state definition these clusters are all semiconducting.

Confidence in the assignment and the existence of delocalized electronic states that are described by a shell model is gained from a comparison of clusters containing atoms from the same group within the periodic table. For $\mathrm{Cu}$ these are $\mathrm{Ag}$ and $\mathrm{Au}$ clusters $/ 10 /$. In such a comparison the alkali's should be included as well, since all these elements have one outer s-electron, which in the solid forms the metallic bond. In Fig. 5 we show some spectra of $\mathrm{Ag}$ and $\mathrm{Na}$ clusters 

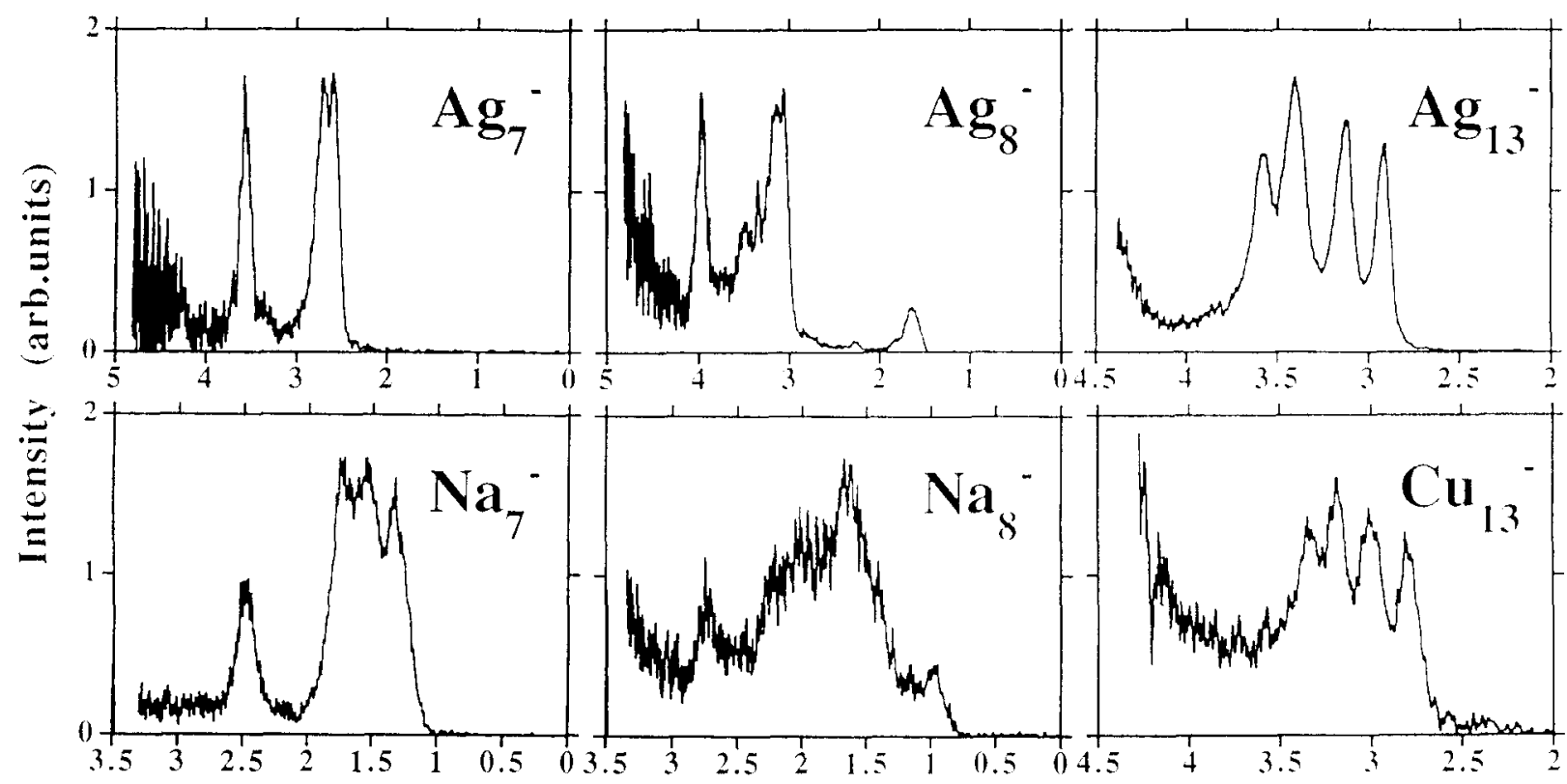

Binding Energy (eV)

Fig. 5 Photoelectron spectra of mass selected $\mathrm{Ag}_{\mathbf{n}}{ }^{-}, \mathrm{Na}_{\mathrm{n}}{ }^{-}$and $\mathrm{Cu}_{13}{ }^{-}$clusters. The spectra of the $\mathrm{Ag}_{\mathrm{n}}{ }^{-}$and $\mathrm{Cu}_{13}{ }^{-}$clusters are recorded with a photon energy of $\mathrm{hv}=5.0 \mathrm{eV}$, the ones of $\mathrm{Na}_{7}{ }^{-}$and $\mathrm{Na}_{8}{ }^{-}$with $\mathrm{hv}=3.49 \mathrm{eV}$.

which may be directly compared with the spectra of the Cu clusters. Na has a much smaller bandwidth than $\mathrm{Cu}$ but contains no atomic d-electrons at all. Accordingly the binding energy scale has been stretched by a factor of 2 in Fig. 5 for the Na clusters. Ag on the other hand, has $10 \mathrm{~d}$-electrons in the atom just as $\mathrm{Cu}$ does, but the $\mathrm{d}$-states of $\mathrm{Ag}$ in the solid have twice the binding energy, measured relative to $\mathrm{EF}_{\mathrm{F}}$, as $\mathrm{Cu}$ d-states. Thus any hybridization between the atomic $s$ and d-states is much less likely to occur for $\mathrm{Ag}$, than for $\mathrm{Cu}$ clusters. Actually, whereas we observed some indications of s-d hybridization in the Cu clusters $/ 6$, we were not able to identify anything comparable in the spectra of Ag clusters.

Comparing the spectra of the 7 and 8 atom clusters of $\mathrm{Cu}, \mathrm{Ag}$, and $\mathrm{Na}$ shown in Figs. 3 and 5 , we notice a remarkable similarity. The $\mathrm{Ag}_{7}^{-}$spectrum shows the emission of three non-degenerate $1 p$ delocalized states, whereas for the $\mathrm{Na}_{7}{ }^{-}$the equivalent $1 \mathrm{p}$ state emission is observed between 1 and $2 \mathrm{eV}$ binding energy and the $1 \mathrm{~s}$ emission is observed at $2.5 \mathrm{eV}$ binding energy. All this points to a full occupation of the 1p shell for the 7 atom cluster-anions. For the 8 atom clusters an additional electron has been added, which now occupies a new state. Within the shell model this is the $1 \mathrm{~d}$ state. Also the spectra of $\mathrm{Cu}_{13}{ }^{-}$and $\mathrm{Ag} 13^{-}$(Fig. 5) are very similar, now predominantly showing emission from $1 \mathrm{~d}$ delocalized states within the range of binding energies displayed here.

For small Ag-clusters containing less than 10 atoms there are quantum-chemical calculations (HF-CI) available, which allow for a more accurate assignment of the states observed in these spectra /11/. With a few exceptions the calculated states agree quite well with the transitions observed in our detachment spectra $/ 7 /$. Moreover, in all these cases, the symmetry of the calculated CI states corresponds to the symmetry of the 
state based upon our assignment within the shell model. This is certainly quite an encouragement for carrying out an assignment of the cluster spectra on the basis of this simple, back-of-the-envelope, shell model. A similar comparison has been carried out by W. Andreoni /12/, who found a large overlap between the electronic state wavefunctions of $\mathrm{Na}$-clusters, calculated by first principles, and the wavefunctions calculated in the shell model.

Obviously not only the VDE's but even more so the complete picture of the electronic states can be used to derive the geometry of the clusters by comparing the electronic structure of the same size cluster, calculated for various geometric configurations, with our spectra. We hope that by providing these spectra experimentally, more theoreticians are encouraged to calculate the electronic structure of clusters for various geometries, maybe even in combination with a molecular dynamics, simulated annealing approach.

\section{B. Chemisorption of small Molecules on Clusters}

Supported metal clusters are the active centers in a large variety of catalysts used in chemical production. The variation in reactivity and selectivity of the supported small particles in the application in a given catalytic process is in general attributed to both a different electronic structure of the small supported particles as well as the existence of unique sites for chemisorption, which are not present at an extended flat surface of the corresponding metal or alloy. A lot of research has been performed to identify and improve the catalytic centers in these systems. Despite of this research the exact microscopic nature of the catalytic centers for most applications still remains unknown.

Mass selected clusters offer the possibility to study chemical reactions with uniquely defined particles. Obviously the hope is to identify some 'new' catalytic properties, which may be conserved by depositing these special clusters onto a suitable substrate. Such catalysts produced containing unique size clusters may enhance the reactivity or even more important the selectivity of the catalytic process.

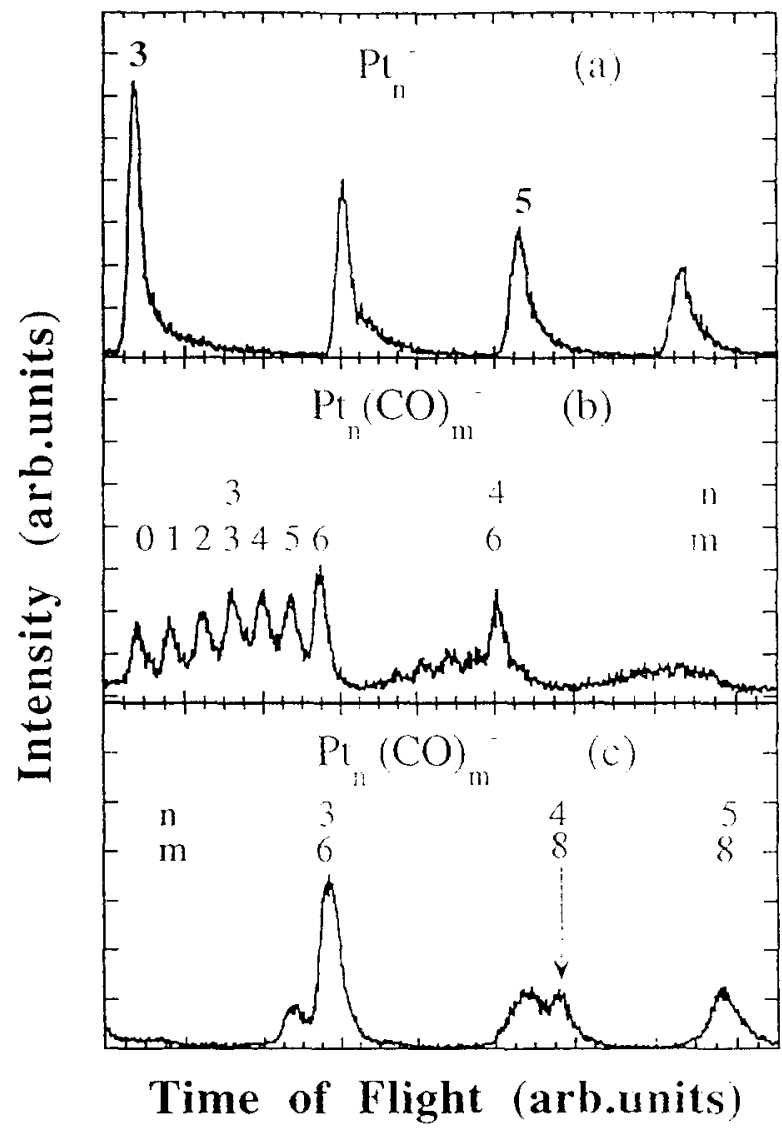

Fig. 6 Time of flight mass spectra of clean $\mathrm{Pt}$ clusters (a), after partial reaction with $\mathrm{CO}(\mathrm{b})$, and after highest exposure possible in our experiments (c). (For more details see text)

Studies of chemical reactions with clusters $/ 13-15 /$ require only a small modification of the experimental setup shown in Fig. 1. A pulsed gas inlet is installed into the high pressure channel where the clusters are already formed and are cooling down drifting towards the expansion zone. At this location a reactant gas may be introduced into the $\mathrm{He}$ atmosphere, which will interact with the clusters. Even though the temperature of the clusters is not too well defined at this point, we have to add the reactant gas in the high pressure zone in order to remove the chemisorption energy through the collisional cooling process. 
Fig. 6 shows some cluster TOF mass spectra of Pt-clusters containing from 3 to 6 Pt-atoms. Adding a small amount of $\mathrm{CO}$ in the high pressure region we observe the mass distribution shown in the center panel. For $\mathrm{Pt}_{3}{ }^{-}$there is a well resolved series of up to 6 chemisorbed $\mathrm{CO}$ molecules per $\mathrm{Pt}_{3}$ cluster detected, whereas $\mathrm{Pt}_{4}{ }^{-}$also shows a prominent peak with 6 chemisorbed $\mathrm{CO}$ molecules. At the highest $\mathrm{CO}$ exposures possible in our experimental setup we observe that the $\mathrm{Pt}_{3}{ }^{-}$clusters exhibit a saturation 'coverage' of $6 \mathrm{CO}$ molecules, whereas $\mathrm{Pt}_{4}{ }^{-}$saturates with $8 \mathrm{CO}$ molecules and $\mathrm{Pt}_{5}{ }^{-}$shows a distribution centered at 8 chemisorbed molecules, which has not yet reached saturation. This is in agreement with the observations reported by Erwin et al. /16/ earlier.

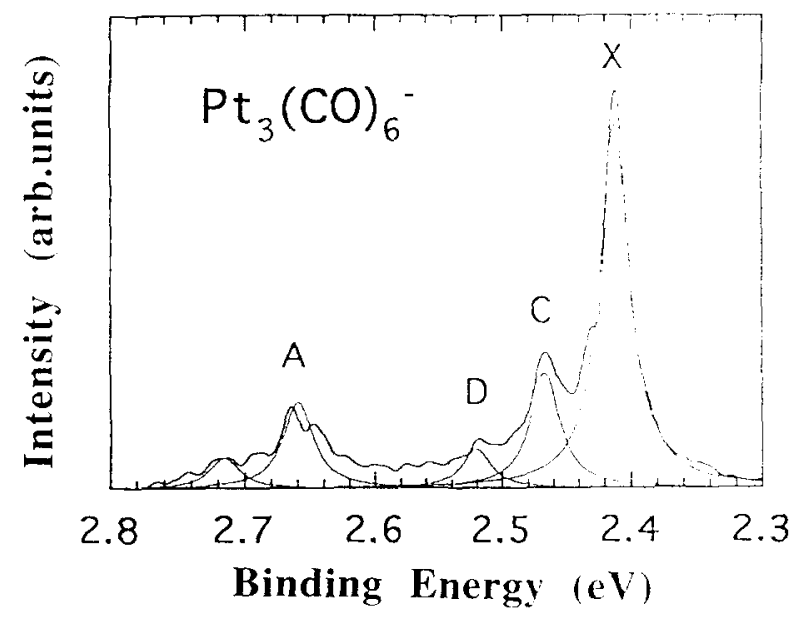

Fig. 7 High resolution photoemission spectrum of $\mathrm{Pt}_{3}(\mathrm{CO})_{6}{ }^{-}$displaying the excitation of different vibrational modes of the cluster. The peak marked $X$ corresponds to the ground state transition. A deconvolution of the spectrum is suggested by the Lorentzians as indicated.

The saturation of the coverage $\mathrm{CO}$ is one of the interesting aspects where clusters deviate a lot from surfaces. On the surface the coverage rarely exceeds one molecular unit per surface atom, whereas for other saturated carbonyl complexes (Fe, Ru) a saturation of $12 \mathrm{CO}$ 's for a three metal atom center is found $/ 17 /$. Since the metal 'core' for all these carbonyls has almost the same triangular geometry, we are inclined to rule out topological hindrance as the reason for the difference in saturation. This suggests that electronic effects are the cause of the saturation in Pt-carbonyls, a proposition which can be checked by photoelectron spectroscopy. For the saturated clusters we indeed observe a pronounced peak at the onset of the photoemission, which exhibits vibrational substructure due to the excitation of both the $\mathrm{CO}$ stretch frequency and, less pronounced, the carbon-metal stretch frequency. A high resolution spectrum of $\mathrm{Pt}_{3}(\mathrm{CO})_{6}{ }^{-}$is shown in Fig. 7. We interpret the sharp emission feature as due to the emission from the antibonding $d-2 \pi$ orbital manifold, which becomes occupied in the negatively charged cluster. These sharp features appear in the spectra of $\mathrm{Pt}_{3}{ }^{-}$and $\mathrm{Pt}_{4}{ }^{-}$clusters only for the CO-saturated clusters, accordingly we believe that saturation is due to a complete filling of the bonding $d-2 \pi$ orbital manifold in the saturated neutral species.

The frequencies observed in the two vibrational progressions associated with the transition into the ground state of the neutral cluster are $2020 \pm 60 \mathrm{~cm}^{-1}$ and $425 \pm 30 \mathrm{~cm}^{-1}$. These are almost the same frequencies as reported for the $\mathrm{CO}$ stretch frequency and the $\mathrm{C}$-metal vibrational frequency for $\mathrm{CO}$ chemisorbed in an 'on-top' site on a Pt surface $/ 18$. The carbonyl has a planar geometry $/ 19 /$, where three CO molecules are bound in 'on-top' and the other three $\mathrm{CO}$ are bound in 'bridge' positions around the triangular $\mathrm{Pt}$ core. Since we observe only the CO-stretch frequency of the ligands in the 'on-top' position, we conclude that the antibonding $d-2 \pi$ orbital of these ligands must be lower in energy than the corresponding molecular orbital of the CO's which are bound in bridge sites.

In summary of these first photoemission studies of chemisorption on mass selected clusters, we could show that the saturation 
of the $\mathrm{CO}$ chemisorption on Pt-clusters is due to an electronic effect rather than due to topological reasons. Judged by the observed vibrational frequencies, however, the chemisorption on these clusters seems to be not much different from chemisorption on the corresponding metal surfaces.

\section{Photoemission of $\mathrm{C}_{60}{ }^{-}$and the superconductivity of the Fullerenes}

As it is well known, the fullerides, which are molecular solids formed by condensation of $\mathrm{C}_{60}$ become superconducting at temperatures exceeding $30 \mathrm{~K}$, when doped with an appropriate amount of alkali atoms $/ 20 \%$. Next to the so called high $\mathrm{T}_{c}$ superconductors, these alkali doped fullerides are thus the superconductors with the highest transition temperatures known so far, and in contrast to the cuprates, they are threedimensional superconductors. Upon alkali doping, electrons are transferred into the $t_{1 u}$ derived band and these electrons are responsible for the metallic and superconducting properties of the solid.

As formulated by Antropov et al. /21/, the coefficients describing the intensity of the vibrational sidebands observed in photoemission of the electrons relevant for the conductivity of any material and the parameters describing the coupling in the superconducting state of this material are only differing by a frequency factor. This seems obvious, if the conductivity is viewed as a hopping process. An electron hopping off a $\mathrm{C}_{60}$ unit in the condensed fullerenes leaves the molecular unit in a vibrationally excited state which lowers the energy barrier for a second electron to move onto this specific $\mathrm{C}_{60}$ unit. Thus an attractive coupling between the two electrons is established.

Since the fullerides are forming molecular solids characterized by a rather weak vander-Waals interaction, the electronic states as well as the intra molecular vibrational modes hardly change at all upon condensation into the solid $/ 22 /$. Also the additional phonon modes of the solid are not contributing much to the superconductivity, even of

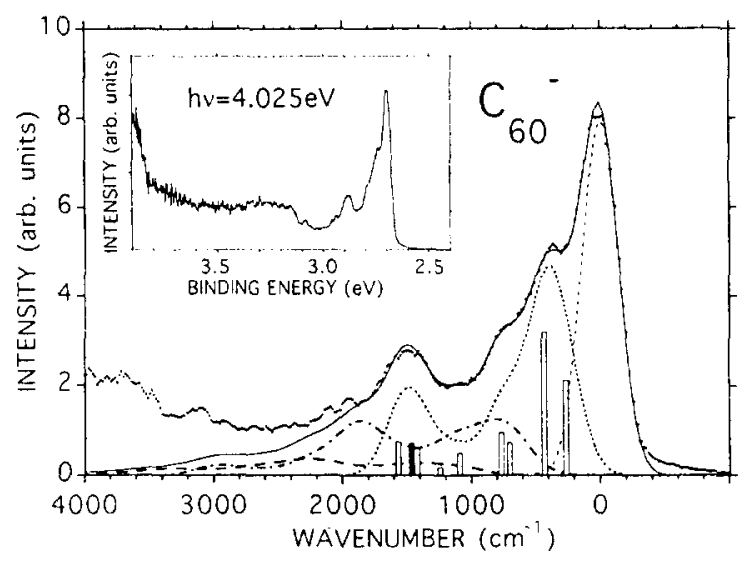

Fig. 8 Experimental spectrum of $\mathrm{C}_{60}{ }^{-}$ photoemission (dots) and theoretical approximation (full line). The theoretical no loss (dashed), single loss (dotted), and triple loss (dash-dotted) curves are shown also [from ref. 20]. The contributions of the various modes to the single loss curve are indicated by the bars $\left(\mathbf{H}_{\mathrm{g}}\right.$ open; $A_{g}$ solid). The inset shows the experimental spectrum over a wider range.

the alkali doped material /21/. Thus photoemission of the $t_{1} u$ orbital of the isolated $\mathrm{C}_{60}{ }^{-}$molecule can reveal the electron phonon coupling strengths relevant for the superconducting state of the alkali doped fullerides.

In Fig. 8 a high resolution photoemission spectrum of $\mathrm{C}_{60^{-}}$is shown, which clearly exhibits finestructure due to vibrational excitations. In the detachment of an electron from the $t_{1 u}$ orbital of $\mathrm{C}_{60}{ }^{-}$altogether $8 \mathrm{H}_{\mathrm{g}}$ and $2 \mathrm{Ag}$ vibrational modes may be excited. The frequencies of these modes are well known from Raman spectroscopy /23/. Using a sophisticated fit procedure, taking into account the Jahn-Teller distortion of the anion and multiple loss features, the relative coupling strengths to the $\mathrm{H}_{\mathrm{g}}$ modes can be extracted from the spectrum shown in Fig. 8. The detailed values of the coupling constants derived from this fit are given elsewhere $/ 20 \%$. In general, the total electron phonon coupling 
is stronger than predicted by theory /21/. Moreover, the relative weight of the coupling is also different. We find that the coupling to the low energy modes, and here mostly the $\mathrm{H}_{\mathrm{g}}(2)$ mode, carries the largest weight, whereas in the calculations the coupling to the high energy $\left(H_{g}(7), H_{g}(8)\right)$ modes dominates. As an overall conclusion, however, the superconductivity of the alkali doped fullerides may be comfortably explained by the conventional BCS theory.

\section{Conclusions}

High resolution electron spectroscopy of mass selected cluster anions reveals information about the electronic structure as well as the geometry of these particles. Thus the evolution of the materials properties of the clusters can be followed step by step as the number of atoms within the cluster is increased. The most important general result of these initial studies is that the properties of the clusters do not develop in a continuous fashion. This leaves ample room for the discovery of interesting new materials.

\section{References}

1. see for example in 'Progress in Fullerene Research'; ed. by $\mathrm{H}$. Kuzmany, J. Fink, M. Mehring, S. Roth, World Scientific, Singapore (1994).

2. Chia-Yen Cha, G. Ganteför, and W. Eberhardt, Rev. Sci. Instrum. 63 (1992). 5661.

3. H. Handschuh, G. Ganteför, and W. Eberhardt, Rev. Sci. Instrum. (1995), in press.

4. P. Kruit and F.H. Read, J. Phys. E16 (1983) 313 .

5. W. de Heer, Rev. Mod. Phys. 65 (1993) 611 , and references therein.
6. Chia-Yen Cha, G. Ganteför, and W. Eberhardt, J. Chem. Phys. 99 (1993) 6308.

7. H. Handschuh, Chia-Yen Cha, P.S. Bechthold, G. Ganteför, and W. Eberhardt, J. Chem. Phys. 102 (1995) 6406.

8. O. Cheshnovsky, K.J. Taylor, J. Conceicao, and R.E. Smalley, Phys. Rev. Lett. 64, (1990) 1785 .

9. Chia-Yen Cha, G. Ganteför, and W. Eberhardt, J. Chem. Phys. 100 (1994) 1.

10. H. Handschuh, Chia-Yen Cha, H. Möller, P.S. Bechthold, G. Ganteför, and W. Eberhardt, Chem. Phys. Lett. 227 (1994) 496.

11. V. Bonacic-Koutecky, L. Cespiva, P. Fantucci, J. Pittner, and J. Koutecky, J. Chem. Phys. 100 (1994) 490.

12. U. Röthlisberger and W. Andreoni, J. Chem. Phys. 94 (1991) 8129.

13. E.K. Parks, L. Zhu, J. Ho, and S.J. Riley, J. Chem. Phys. 100 (1996) 7206.

14. B.C. Guo, K.P. Kerns, and A.W Castleman, J. Chem. Phys. 96 (1992) 8177.

15. D.M. Cox, K.C. Reichmann, D.J. Trevor, and A. Kaldor, J. Chem. Phys. 88 (1988) 111.

16. P.A. Hintz and K.M. Ervin, J. Chem. Phys. 100 (1994) 5715.

17. see, e.g., E.W. Plummer, W.R. Salaneck, and J.S. Miller, Phys. Rev. B 18 (1978) 1673.

18. F.M. Hoffmann, Surf. Sci. Reports. 3 (1978) 107.

19. G. Longoni and P. Chini, J. Am. Chem. Soc. 98 (1976) 7225.

20. O. Gunnarsson, H. Handschuh, P.S. Bechthold, B. Kessler, G. Ganteför, and W. Eberhardt, Phys. Rev. Lett. 74 (1995) 1875, and references therein.

21. V.P. Antropov, O. Gunnarsson, and A.I. Liechtenstein, Phys. Rev. B 48 (1993) 7551.

22. S. Krummacher, M. Biermann, M. Neeb, A. Liebsch, and W. Eberhardt, Phys. Rev. B 48 (1993) 8424.

23. D.E. Bethune, G. Meijer, W.C. Tang, H.J. Rosen, W.G. Golden, H. Seki, C.A. Brown, and M.S. de Vries, Chem. Phys. Lett. 179 (1991) 181. 